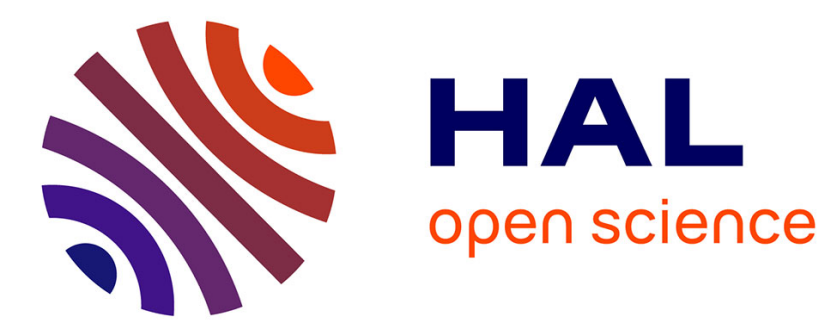

\title{
Parameter Estimation of a Mathematical Model Describing the Cardiovascular-Respiratory Interaction
}

Layli S. Goldoozian, Antonio Hidalgo-Muñoz, Vicente Zarzoso, Edmond Zahedi

\section{- To cite this version:}

Layli S. Goldoozian, Antonio Hidalgo-Muñoz, Vicente Zarzoso, Edmond Zahedi. Parameter Estimation of a Mathematical Model Describing the Cardiovascular-Respiratory Interaction. Computing in Cardiology, 42nd Annual Conference, Sep 2015, Nice, France. 10.1109/CIC.2015.7410986 . hal01302628

\section{HAL Id: hal-01302628 \\ https://hal.science/hal-01302628}

Submitted on 14 Apr 2016

HAL is a multi-disciplinary open access archive for the deposit and dissemination of scientific research documents, whether they are published or not. The documents may come from teaching and research institutions in France or abroad, or from public or private research centers.
L'archive ouverte pluridisciplinaire HAL, est destinée au dépôt et à la diffusion de documents scientifiques de niveau recherche, publiés ou non, émanant des établissements d'enseignement et de recherche français ou étrangers, des laboratoires publics ou privés. 


\title{
Parameter Estimation of a Mathematical Model Describing the Cardiovascular-Respiratory Interaction
}

\author{
Layli S. Goldoozian ${ }^{1}$, Antonio R. Hidalgo-Muñoz ${ }^{2}$, Vicente Zarzoso ${ }^{2}$, Edmond Zahedi ${ }^{1,3}$ \\ ${ }^{1}$ School of Electrical Engineering, Sharif University of Technology, Tehran, Iran \\ ${ }^{2}$ Laboratoire I3S, Université Nice Sophia Antipolis, CNRS, France \\ ${ }^{3}$ Department of Electronics, Electrical \& System Engineering, Faculty of Engineering and built-in \\ Environment, National University Malaysia (Universiti Kebangsaan Malaysia)
}

\begin{abstract}
Short-term interaction between heart rate $(H R)$ and physiological measures like blood pressure and respiration reveals relevant information about autonomic nervous system (ANS) function. Complex mathematical models for describing their couplings have been proposed in the literature. However, an accurate estimation of their parameters in an inverse modeling problem is crucial to extract reliable ANS related indices. This study considers a physiologically-based model of the cardiovascularrespiratory system and ANS control that presents the neural and mechanical effects of respiration separately. The estimation method is evaluated on synthetic signals. An accurate estimation of the highest-sensitivity model parameter (intrinsic HR) is achieved with an error of $4.7 \pm$ $3.4 \%$ over the actual values. One of the parameters reflecting the amplitude of the respiratory-mediated variations presents an even better approximation with a mean relative error as low as $3.8 \pm 3.3 \%$. Our results show that most of the high-sensitivity parameters and also respiratoryrelated parameters that are specifically considered in our physiologically-based framework can be well approximated regardless of their initial values.
\end{abstract}

\section{Introduction}

Cardiovascular regulation over short time scales (of seconds to minutes) is one of the most important functions of the autonomic nervous system (ANS) that is mediated via parasympathetic and sympathetic nerves [1]. Therefore, short-term variations in some cardiovascular measures like heart rate (HR) and blood pressure (BP) are commonly

L. Goldzoozian is in part supported by a grant from the Embassy of France in Tehran.

A. Hidalgo is in part supported by a Postdoctoral Fellowship from the University of Nice Sophia Antipolis.

V. Zarzoso is a member of the Institut Universitaire de France. studied to assess ANS function. In practice, an external stimulation perturbing ANS, e.g., sit-to-stand or respiratory maneuvers, is applied and ANS-related indices are extracted from the BP or HR (or their relative change). Although there are some clinical methods for the evaluation of ANS [2], there is still the need to assess its function in an online and reliable way.

Respiration causes fluctuations in cardiovascular parameters like HR and BP through both ANS and mechanical paths [3]. HR variability due to respiration is known as respiratory sinus arrhythmia (RSA), and its analysis is used for assessing autonomic health. Some studies have considered complex models to explain the joint relation between the cardiovascular signals and respiration $[4,5]$. However, estimating such model parameters reliably is of great importance so that they may be applied as time-varying biomarkers of ANS function [6].

The present work is based on a joint model of the cardiovascular-respiratory system and ANS control to simulate the short-term relations between HR, BP and respiration [7]. The efficiency of the parameter estimation method is specifically studied here. Inverse modeling is done in two steps. Firstly, a sensitivity analysis is performed to define the most significant parameters of the model. Secondly, the parameters are estimated using well-known optimization algorithms (Nelder-Mead). Finally, the accuracy of the parameter estimation method is assessed on synthetically generated data.

\section{Modelling}

\subsection{Cardiovascular-baroreflex interaction}

A 2-element Windkessel model consisting of the total arterial resistance and arterial compliance has been considered for the cardiovascular system [8]:

$$
\dot{P}(t)=-\frac{P(t)}{R_{c} C_{a}}+\frac{H(t) \Delta V}{C_{a}}
$$


where $H(t)$ is the instantaneous $\mathrm{HR}, P(t)$ the mean BP, $R_{c}$ and $C_{a}$ the total arterial resistance and compliance respectively, and $\Delta \mathrm{V}$ the stroke volume.

The baroreflex system, as an important part of the ANS, aims at maintaining BP in a narrow physiological range. $\mathrm{BP}$ is sensed by the baroreceptors which transmit afferent signals to the brain. The brain sends some efferent signals back to the cardiovascular parts via sympathetic and parasympathetic nerves where BP regulation is done by adjusting some physiological parameters like HR, ventricular contractility, arterial resistance and systematic venous unstressed volume. The models considered for baroreflex control of HR and arterial resistance [8], are given respectively by:

$$
\begin{gathered}
\dot{H}(t)=\frac{\beta_{H} T_{s}}{1+\gamma T_{p}}-V_{H} T_{p}+\delta_{H}\left(H_{0}-H(t)\right) \\
R_{c}=R_{c}^{0}\left(1+\alpha T_{s}\right)
\end{gathered}
$$

where $T_{p}$ and $T_{s}$ are the parasympathetic and sympathetic tones respectively which can be modeled as sigmoidal functions of pressure:

$$
\begin{aligned}
& T_{s}(t)=\frac{1}{1+\left(P(t-\tau) / P_{0}\right)^{r}} \\
& T_{p}(t)=1-\frac{1}{1+\left(P(t) / P_{0}\right)^{r}}
\end{aligned}
$$

The definitions and nominal values [8] of the parameters in eqns. (1)-(5) are presented in Table 1.

Table 1. Model parameter definitions and nominal values [8]

\begin{tabular}{lll}
\hline \hline Parameter & Description & Value \\
\hline$C_{a}$ & arterial compliance & $50 \mathrm{ml} . \mathrm{mm} \cdot \mathrm{Hg}^{-1}$ \\
$R_{c}^{0}$ & min arterial resistance & $0.6 \mathrm{mmHg} . \mathrm{s} . \mathrm{ml}^{-1}$ \\
$\beta_{H}$ & sympathetic control of HR & $0.84 \mathrm{~s}^{-2}$ \\
$\gamma$ & vagal damping of $\beta_{H}$ & 0.2 \\
$V_{H}$ & vagal control of HR & $1.17 \mathrm{~s}^{-2}$ \\
$\delta_{H}$ & inverse relaxation time & $1.7 \mathrm{~s}^{-1}$ \\
$H_{0}$ & uncontrolled HR & $100 \mathrm{bps}$ \\
$\Delta V$ & stroke volume & $50 \mathrm{ml}$ \\
$\tau$ & sympathetic delay & $3 \mathrm{~s}$ \\
$\alpha$ & sympathetic effect on $R_{c}$ & 1.3 \\
$P_{0}$ & mean arterial pressure & $93 \mathrm{mmHg}$ \\
\hline
\end{tabular}

\subsection{Respiratory effects}

As mentioned, respiration affects cardiovascular system through mechanical and neural paths. HR increases and decreases during inspiration and expiration respectively. A direct neural coupling exists between respiratory system and heart. This neural mechanism affects HR mostly via respiratory related variations in the power $\left(V_{H}\right)$ of the parasympathetic tone $\left(T_{p}\right)$ [9]. Therefore, eqn. (2) is modified as below:

$$
\dot{H}(t)=\frac{\beta_{H} T_{s}}{1+\gamma T_{p}}-\left(V_{H}-k_{1} V_{L}(t)\right) T_{p}+\delta_{H}\left(H_{0}-H(t)\right)
$$

where $V_{L}(t)$ is the instantaneous lung volume and $k_{1}$ is the factor of lung volume in modulating $V_{H}$.

The mechanical effect of respiration on the cardiovascular system should be reflected in the model by respiratory modulated changes in the stroke volume, which itself happens as a result of respiratory-synchronous variations in the intra-thoracic pressure $[7,9]$. This effect is incorporated in eqn. (1) as:

$$
\dot{P}(t)=-\frac{P(t)}{R_{c} C_{a}}+\frac{H(t)\left(\Delta V-k_{2} V_{L}(t)\right)}{C_{a}}
$$

where the coefficient $k_{2}$ is intended to adjust the effect of $V_{L}$ in modulating the stroke volume.

As can be perceived from eqn. (7), modulation of $\Delta V$ by respiration leads to respiratory-related variations in the pressure. Consequently, in addition to the direct neural path, HR is also indirectly modulated by the respiration via the link between HR and BP (baroreflex control). Remark that parameters $k_{1}$ and $k_{2}$ in eqns. (6)-(7) are related to the gains of the respiratory-related variations of the outputs.

\section{Inverse modeling}

\subsection{Sensitivity analysis}

Prior to the estimation of the model parameters, it is essential to define the parameters to which the model outputs have higher sensitivity. Sensitive parameters are indeed those whose variations cause significant changes in the outputs [6]. The sensitivity analysis was carried out at nominal parameter values. The instantaneous normalized sensitivity of each of the outputs (in our case, HR or pressure in eqns. (6) and (7) respectively) to the $i$-th parameter $\theta_{i}$ at time instant $t$ is calculated as:

$$
S_{n, i}(t)=\left.\frac{\partial y_{n}(t, \theta)}{\partial \theta_{i}} \frac{\theta_{i}}{y_{n}}\right|_{\theta_{i}=\theta_{i 0}}
$$

where $\theta_{i 0}$ denotes the nominal value of parameter $\theta_{i}$ and $y$ stands for the model output; the subscript $n$ refers to either HR or BP. The total sensitivity index of the model to $\theta_{i}$ is defined as the scaled 2-norm of the instantaneous sensitivity over the two outputs:

$$
S_{i}=\sqrt{\frac{1}{2 m} \sum_{n, t} S_{n, i}^{2}(t)}
$$

where $m$ is the number of time samples over which the sensitivity is calculated for each output. Parameter values are split into high-sensitivity and low-sensitivity groups according to their total sensitivity values $S_{i}$. 


\subsection{Parameter estimation}

Having defined the sensitivity of the model to the parameters, the parameters of the high-sensitivity group are estimated in the model identification process while fixing the value of the other parameters at their nominal values (Table 1). The parameter identification was carried out in two steps. Firstly, all high-sensitivity parameters were estimated such as to minimize the normalized mean squared error between the model outputs and the data.

At the second step, the respiratory-related parameters $k_{1}$ and $k_{2}$ were estimated while fixing the values of the other parameters at their estimated values obtained from the first step or at their nominal values for the low-sensitivity parameters. Oscillations at respiratory rate range are often referred to as high-frequency oscillations. Since respiration is only expected to introduce high-frequency fluctuations in the model outputs, the cost function to be minimized at this step was the normalized mean squared error between the instantaneous amplitude of the model outputs and the actual data:

$$
E=\frac{1}{2 m} \sum_{n, t}\left(\frac{A m p_{n}^{m o}(t)-A m p_{n}^{s y n}(t)}{A m p_{n}^{s y n}(t)}\right)^{2}
$$

where $A m p(t)$ is the amplitude, and superscripts ' $m o$ ' and 'syn' stand for the model output and actual (synthesized) data, respectively.

To assess the amplitude signals, a peak detection algorithm was applied on the BP or HR signals to find their maxima and minima at each respiratory cycle. The upper and lower envelopes of the signals were defined by spline interpolation, which converts the detected extremum series into evenly spaced signals (Fig. 1). The difference of the upper and lower envelopes was calculated as for the instantaneous amplitude signal.

For both of the parameter identification steps, the Nelder-Mead algorithm (a gradient-free method) in the MATLAB Optimization Toolbox was used to optimize the parameters [10]. The accuracy of the estimation for each parameter was defined as the percent error between the actual and the estimated values of the parameter averaged over 100 trials.

\subsection{Evaluation}

The accuracy of the parameter identification method was assessed on the synthesized data. One hundred data segments of 30-second length were generated by random perturbations of the model parameters of up to $30 \%$ of their nominal values. The model outputs were sampled at a frequency of $5 \mathrm{~Hz}$. As instantaneous respiratory signal $V_{L}(t)$, which is an input to the model, we employed a recorded respiratory signal obtained from a MIMIC dataset [11].

\section{4. $\quad$ Results}

\subsection{Sensitivity analysis}

The result of the sensitivity analysis for the model is shown in Fig. 2. Parameters $\left\{H_{0}, \Delta V, P_{0}, R_{c}^{0}, \alpha\right\}$ yield the highest sensitivity index values, and are thus classified in the high-sensitivity group, while the rest are considered as low-sensitivity.

\subsection{Parameter estimation and evaluation}

The estimation error for the high-sensitivity parameters determined in the first step is presented in Table 2. Also given are the estimation error of respiratory-related parameters $k_{1}$ and $k_{2}$ when estimated along with the highsensitivity parameters ("s1") and when computed at the second step ("s2") as described in Sec. 3.2.

Table 2. Parameter estimation errors

\begin{tabular}{|c||c|c||c|}
\hline $\begin{array}{c}\text { Parameter } \\
\text { (High- } \\
\text { sensitivity) }\end{array}$ & \multicolumn{1}{|c|}{ Error } & $\begin{array}{c}\text { Parameter } \\
\text { (Respiratory- } \\
\text { related) }\end{array}$ & \multicolumn{1}{|c|}{ Error } \\
$($ Mean $\pm S D) \%$
\end{tabular}

s1: step one, s2: step 2 of parameter estimation

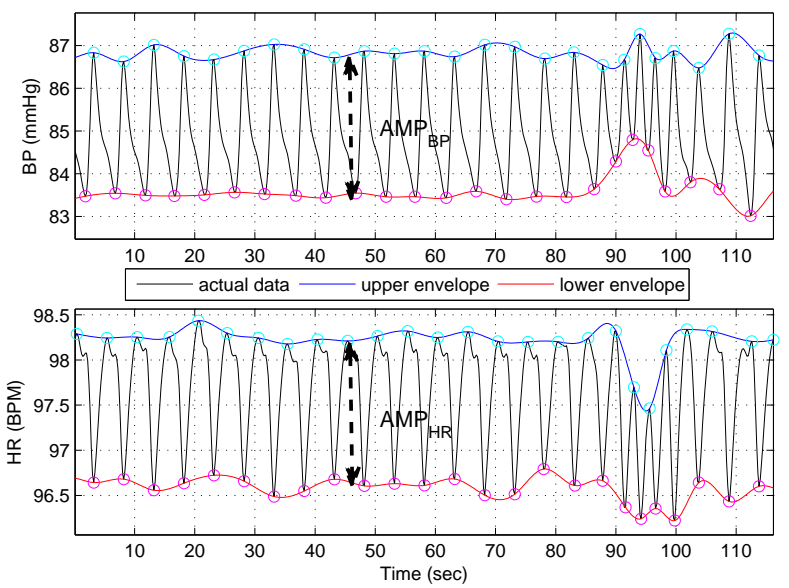

Figure 1. Extracting the upper and lower envelopes of the model outputs, giving rise to the amplitude signals $A m p_{n}^{m o}(t)$ employed in eqn. (10) for the estimation of respiratory-related parameters. Top: BP signal. Bottom: HR signal. The synthesized data amplitudes $A m p_{n}^{s y n}(t)$ are computed in the same way. 


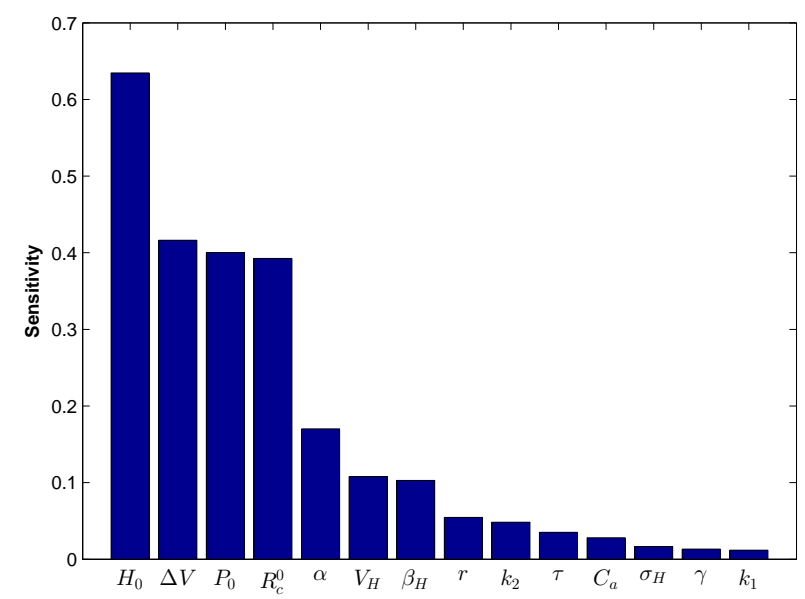

Figure 2. Sensitivity analysis of the model parameters.

\section{Discussion}

Our work has determined the most sensitive parameters in our cardiorespiratory-ANS model and the influence of respiratory-related parameters on the high-frequency oscillations of the model outputs. Parameter estimation results (Table 2) reveal that parameter $H_{0}$ (intrinsic HR), the most sensitive according to the analysis of Fig. 2, can be estimated with a low error of $4.7 \pm 3.4 \%$. Furthermore, the estimation error for most of the other high-sensitivity parameters is relatively low and comparable to the literature. According to the sensitivity analysis (Fig. 2), the parameters $\left\{V_{H}, \beta_{H}, r, \tau, C_{a}, \sigma_{H}, \gamma, k_{1}, k_{2}\right\}$ were classified in the low-sensitivity group. This low-sensitivity group includes parameters $k_{1}$ and $k_{2}$, the gains of the control paths of respiration on the cardiovascular model. As we are specifically interested in the respiratory mediated control of ANS, it is of much importance to estimate these two parameters reliably. The low sensitivity of these respiratory-related parameters causes significant estimation inaccuracies. To compensate for this shortcoming, the second step of parameter estimation was carried out. As reported in Table 2 , the estimation errors improve considerably in the second step, especially for parameter $k_{2}$ (error of $3.8 \pm 3.3 \%$ vs. $8.6 \pm 5.8 \%$ ) in the second step. This is due to the different cost function considered in this step, where the optimization algorithm aims at minimizing the mean squared error between the amplitude of high-frequency (respiratory rate range) oscillations of the model outputs and the data [eqn. (10)]. This cost function was chosen regarding the actual effects of these two parameters which impact the high-frequency components of the outputs more significantly than the low-frequency one, i.e., the high-frequency terms of the signals are more sensitive to these two parameters.

In conclusion, our results show that most model parameters including the respiratory-related ones can be estimated with low error in the framework of our physiologicallybased model. Further work should consider the application of our parameter estimation method to assess reliable online ANS indices in real data.

\section{References}

[1] Xiao X, Mullen TJ, Mukkamala R. System identification: a multi-signal approach for probing neural cardiovascular regulation. Physiological measurement 2005;26(3):R41.

[2] Low PA. Testing the autonomic nervous system. Seminars in neurology 2003;23(4):407-421.

[3] Berntson GG, Cacioppo JT, Quigley KS. Respiratory sinus arrhythmia: autonomic origins, physiological mechanisms, and psychophysiological implications. Psychophysiology 1993;30(2):183-196.

[4] Mullen TJ, Appel ML, Mukkamala R, Mathias JM, Cohen RJ. System identification of closed-loop cardiovascular control: effects of posture and autonomic blockade. American Journal of Physiology Heart and Circulatory Physiology 1997;272(1):H448-H461.

[5] Lu K, Clark J, Ghorbel F, Ware D, Bidani A. A human cardiopulmonary system model applied to the analysis of the valsalva maneuver. American Journal of Physiology Heart and Circulatory Physiology 2001;281(6):H2661-H2679.

[6] Ottesen JT, Novak V, Olufsen MS. Development of patient specific cardiovascular models predicting dynamics in response to orthostatic stress challenges. In Mathematical Modeling and Validation in Physiology. Springer, 2013; 177-213.

[7] Goldoozian LS, Zahedi E. Mathematical modeling of heart rate and blood pressure variations due to changes in breathing pattern. In 20th Iranian Conference on Biomedical Engineering (ICBME). IEEE, 2013; 54-58.

[8] Fowler A, McGuinness M. A delay recruitment model of the cardiovascular control system. Journal of Mathematical Biology 2005;51(5):508-526.

[9] Ataee P, Belingard L, Dumont G, Noubari HA, Boyce WT, et al. Autonomic-cardiorespiratory regulation: A physiology-based mathematical model. In Annual International Conference of the IEEE Engineering in Medicine and Biology Society (EMBC. IEEE, 2012; 3805-3808.

[10] MATLAB Optimization Toolbox Users Guide. MathWorks, Natick, MA, USA, 2013.

[11] Goldberger AL, Amaral LA, Glass L, Hausdorff JM, Ivanov PC, Mark RG, Mietus JE, Moody GB, Peng CK, Stanley HE. Physiobank, physiotoolkit, and physionet components of a new research resource for complex physiologic signals. Circulation 2000;101(23):e215-e220.

Address for correspondence:

Layli Goldoozian

BME Laboratory, Department of Electrical Engineering, Sharif University of Technology

Tehran, Iran

lgoldoozian@ee.sharif.edu 